\title{
O retorno ao trabalho na perspectiva de terapeutas ocupacionais: facilitadores e barreiras*
}

\section{Return to work in the perspective of occupational therapists: facilitators and barriers}

\author{
Talita Naiara Rossi da Silva ${ }^{1}$, Gisele Beatriz de Oliveira Alves ${ }^{2}$, \\ Marcella Guimarães Assis $^{3}$
}

http://dx.doi.org/10.11606/issn.2238-6149.v27i2p116-122

Silva TNR, Alves GBO, Assis MG. O retorno ao trabalho na perspectiva de terapeutas ocupacionais: facilitadores e barreiras. Rev Ter Ocup Univ São Paulo. 2016 maio-ago.;27(2):116-22.

RESUMO: Este estudo tem como objetivo compreender as percepções dos terapeutas ocupacionais em relação aos fatores facilitadores e as barreiras para o retorno ao trabalho. Fundamentase na abordagem qualitativa. Participaram cinco terapeutas ocupacionais que atuam no Instituto Nacional de Seguro Social da Região Metropolitana de Belo Horizonte. A coleta de dados foi realizada por meio de questionário online. As respostas foram analisadas a partir da análise de conteúdo da qual emergiram três categorias temáticas: Segurados: aspectos pessoais e interrelacionais; Empresas e INSS: fatores organizacionais e recursos humanos; e Políticas Públicas: capacitação e contratação de trabalhadores. Os resultados apontaram entre os facilitadores para o retorno ao trabalho a motivação dos trabalhadores, a qualificação, a integração entre INSS e as empresas, e as políticas que apoiam a capacitação. Como barreiras foram reveladas as experiências profissionais restritas, a desarticulação entre as áreas do INSS, o desinteresse das empresas para adaptar situações de trabalho e a descontinuidade dos recursos para a Reabilitação Profissional. O retorno ao trabalho é um processo multifatorial e requer a atuação de diferentes atores sociais na transformação do trabalho para potencializar os facilitadores e modificar as barreiras.

DESCRITORES: Saúde do trabalhador; Retorno ao trabalho; Terapia ocupacional; Previdência social.
Silva TNR, Alves GBO, Assis MG. Return to work in the perspective of occupactional therapists: facilitators and barriers. Rev Ter Ocup Univ São Paulo. 2016 May-Aug.;27(2):116-22.

ABSTRACT: This study aims to understand the perceptions of occupational therapists in relation to facilitators and barriers to return to work. It is based on a qualitative approach. Five occupational therapists who work at the National Institute of Social Security in the metropolitan region of Belo Horizonte have participated. Data were collected through an on-line questionnaire. The responses were analyzed from the content analysis, from which three thematic categories emerged: Insured persons: personal and inter-relational aspects; Enterprises and Social Security: organizational factors and human resources; and Public Policies: training and hiring workers. The results showed between the facilitators for the return to work the workers' motivation, qualification, integration between Social Security and the companies, and the policies that support training. As barriers, the restricted professional experiences, the disarticulation between the Social Security areas, the lack of interest of companies to adapt work situations, and the discontinuity of resources for professional rehabilitation were revealed. The return to work is a multifactorial process and requires the performance of different social actors in the transformation of work to empower facilitators and change barriers.

KEYWORDS: Occupational health; Return to work; Occupational therapy; Social security.

*O artigo é resultado do projeto de pesquisa O processo de retorno ao trabalho dos trabalhadores segurados pelo INSS: a perspectiva dos terapeutas ocupacionais.

1. Terapeuta Ocupacional. Professor Assistente do Departamento de Terapia Ocupacional da Universidade Federal de Minas Gerais. E-mail: talitarossi@ufmg.br

2. Terapeuta Ocupacional. Professor Assistente do Departamento de Terapia Ocupacional da Universidade Federal de Minas Gerais. E-mail: giseleal@eeffto.ufmg.br

3. Terapeuta Ocupacional. Professor Titular do Departamento de Terapia Ocupacional da Universidade Federal de Minas Gerais. E-mail: mga@.ufmg.br

Endereço para correspondência: Universidade Federal de Minas Gerais - Escola de Educação Física, Fisioterapia e Terapia Ocupacional - Departamento de Terapia Ocupacional. Avenida Presidente Antônio Carlos, 6627, Campus Pampulha - Belo Horizonte, MG. CEP: $31270-901$. 


\section{INTRODUÇÃO}

$\mathrm{O}$ trabalho constitui-se como objeto de estudo de diversas disciplinas, é complexo, multidimensional e envolve uma noção abstrata e uma variedade de experiências singulares. Para a ergonomia, o trabalho é uma atividade exercida por homens e mulheres e que media a relação entre o indivíduo e a sociedade ${ }^{1}$. De acordo com Tersac e Maggi ${ }^{1}$ o trabalho é uma ação finalizada que ocorre em um contexto estruturado por dispositivos, regras e normas. Estes autores salientam que o trabalho é cada vez menos compreendido como uma realidade dada, pois requer a mobilização coletiva dos sujeitos para obtenção dos resultados.

Guérin et al. $^{2}$ entendem o trabalho como unidade constituída pelas condições de trabalho, a atividade de trabalho e os resultados do trabalho, que não existem independentemente uns dos outros. O trabalho não é simplesmente a execução de prescrições e envolve a ação dos sujeitos para administrar as contradições que se revelam na articulação entre a dimensão pessoal e a socioeconômica do trabalho.

Nas últimas quatro décadas transformações no contexto socioeconômico modificaram a vida dos indivíduos dentro e fora do trabalho. No contexto atual, ressalta-se a intensificação das atividades de trabalho, a sobrecarga física e mental, a pressão temporal, a gestão por resultados, a elevada responsabilização e a falta de reconhecimento dos trabalhadores. Os desequilíbrios resultantes entre as demandas das empresas aos trabalhadores e os recursos e condições a eles disponibilizadas, contribuem para a constituição de situações estressantes que levam a formas diversificadas de adoecimento e aos acidentes de trabalho ${ }^{3}$. Por conseguinte, colocam desafios para a Reabilitação Profissional ${ }^{4}$.

A reabilitação profissional, segundo Bregalda e Lopes ${ }^{5}$, é um "campo de estudo, produção de conhecimento e desenvolvimento de proposições e ações que remetem aos processos de retorno ao trabalho de indivíduos que, por uma multiplicidade de razões, necessitam de intervenções para a retomada de sua vida profissional" (p. 294). As autoras enfatizam o caráter técnico/político, coletivo/individual das intervenções.

No Brasil, de acordo com o Decreto n 3048/19996, a reabilitação profissional é uma atribuição do Ministério da Previdência Social, realizada pelo Instituto Nacional do Seguro Social (INSS) e "visa proporcionar aos beneficiários incapacitados parcial ou totalmente para o trabalho, em caráter obrigatório, independente de carência, e às pessoas portadoras de deficiência, os meios indicados para o reingresso no mercado de trabalho e no contexto em que vivem".

A reabilitação profissional é desenvolvida por meio de avaliação do potencial laborativo do segurado(1), orientação e acompanhamento da programação profissional, articulação com a comunidade para parcerias e convênios visando o reingresso no mercado de trabalho e acompanhamento e pesquisa da fixação no mercado de trabalho (Art. 137) ${ }^{6}$. A equipe da Reabilitação Profissional é composta por médicos peritos e orientadores profissionais que incluem fisioterapeutas, terapeutas ocupacionais, pedagogos, psicólogos, sociólogos, assistentes sociais e outros ${ }^{4}$.

Segundo dados da Previdência Social no período de 2011 a $2013^{7}$ foram registrados 2.152 .524 acidentes de trabalho $^{(2)}$ que resultaram em incapacidades temporárias e permanentes. Desse total, 157.980 segurados foram encaminhados para a Reabilitação Profissional. Após a avaliação inicial 11.440 segurados retornaram à atividade de trabalho, 32.740 foram considerados inelegíveis e 96.797 elegíveis para a Reabilitação Profissional. No mesmo período o Estado de Minas Gerais registrou 232.865 acidentes de trabalho, dos quais 13.351 segurados foram registrados na Reabilitação Profissional. Após a avaliação inicial, 846 segurados retornaram para o trabalho, 2.775 foram considerados inelegíveis para a Reabilitação e 8.498 elegíveis. Entre os segurados registrados na Reabilitação Profissional foram considerados elegíveis $61 \%$ no âmbito nacional e $63 \%$ no Estado de Minas Gerais, o que sinaliza, por um lado, uma elevada porcentagem de encaminhamentos para a Reabilitação e, por outro, a necessidade de conhecer os desfechos no que diz respeito ao retorno ao trabalho.

A definição de retorno ao trabalho não é consensual e existem diferentes modelos conceituais ${ }^{8}$. Young et al. ${ }^{9}$ definem o retorno ao trabalho como fenômeno que abrange tanto o processo quanto os resultados associados. Em relação ao processo os autores apontam diferentes fases: 1) afastamento do trabalho (off work) devido a descoberta

(1) Segurado: termo que refere-se aos trabalhadores que são contribuintes do INSS e assegurados pela previdência social.

(2) A Previdência Social ${ }^{7}$ considera como acidente de trabalho a doença profissional e a doença do trabalho; o acidente ligado ao trabalho que, embora não tenha sido a causa única, haja contribuído diretamente para a ocorrência da lesão; certos acidentes sofridos pelo segurado no local e no horário de trabalho; a doença proveniente de contaminação acidental do empregado no exercício de sua atividade; e o acidente sofrido a serviço da empresa ou no trajeto entre a residência e o local de trabalho do segurado e vice-versa. 
de uma condição funcional; 2) fase de recomeço (re-entry) do trabalho na mesma atividade/local anterior a lesão ou outra; 3) manutenção do desempenho (maintenance), o trabalhador consegue realizar a atividade de acordo com os objetivos esperados e; 4) progressão no trabalho (advancement), quando o trabalhador tem um desempenho consistente e busca avançar nas responsabilidades, qualificação e carreira. O percurso de cada trabalhador entre estas fases não é linear. Este modelo mostra que o retorno ao trabalho é um fenômeno dinâmico e multifatorial ${ }^{10} \mathrm{e}$ requer a articulação de ações de diferentes atores sociais ${ }^{11}$.

$\mathrm{O}$ afastamento do trabalho pode levar ao redimensionamento da participação do indivíduo nas atividades e papéis exercidos cotidianamente ${ }^{12}$. O terapeuta ocupacional pode contribuir para o retorno ao trabalho por meio de ações de prevenção de novos agravos, avaliação e acompanhamento do trabalhador e da situação de trabalho ${ }^{13}$.

$\mathrm{Na}$ perspectiva dos terapeutas ocupacionais, as principais barreiras para o retorno ao trabalho, identificadas a partir da atuação em agências do INSS do Estado de São Paulo, são: medo de demissão após o retorno à empresa ou término do período de estabilidade, a baixa escolaridade, longos períodos de afastamento, a comodidade trazida pelo recebimento do benefício e, a ausência de motivação para se capacitar profissionalmente em outras áreas ${ }^{5}$.

A análise de registros de prontuários de trabalhadores atendidos em um Centro de Referência em Saúde do Trabalhador (CRST), do município de São Paulo, revelou como barreiras as limitações funcionais e os obstáculos vivenciados junto às empresas, à previdência social e aos serviços de saúde e, como facilitadores, os apoios, as atitudes individuais e os relacionamentos com os profissionais da saúde ${ }^{4}$.

Considerando a necessidade de ampliação do conhecimento sobre o retorno ao trabalho, este estudo objetiva compreender as percepções dos terapeutas ocupacionais, orientadores profissionais no INSS da Região Metropolitana de Belo Horizonte, em relação aos fatores facilitadores e as barreiras para o retorno dos segurados ao trabalho.

\section{MÉTODO}

O estudo baseou-se na metodologia qualitativa ${ }^{14}$. Contou com a participação de terapeutas ocupacionais que atuavam nas Agências do INSS da Região Metropolitana de Belo Horizonte ${ }^{(3)}$, conforme relação fornecida pela Superintendência Regional.

No período compreendido entre abril e agosto de 2015 foi realizado contato telefônico com os 10 profissionais. A participação foi voluntária e definida após a apresentação do projeto e concordância registrada no Termo de Consentimento Livre e Esclarecido (TCLE).

Para coleta de dados utilizou-se um questionário online elaborado pelos pesquisadores. $\mathrm{O}$ respectivo link de acesso foi enviado aos participantes do estudo via e-mail. O questionário abordou questões abertas relacionadas às seguintes temáticas: retorno dos segurados ao trabalho, atuação dos terapeutas ocupacionais/ orientadores profissionais na Reabilitação Profissional, fatores facilitadores e barreiras observados por estes profissionais; além de dados sociodemográficos como idade, escolaridade, função e tempo de atuação no INSS.

Os dados coletados foram analisados utilizandose a análise de conteúdo temática ${ }^{16}$, a qual consistiu de três etapas: leituras flutuantes e exploratórias do texto, exploração do material e formação de categorias analíticas e tratamento dos resultados obtidos e interpretação.

$\mathrm{O}$ projeto de pesquisa foi submetido e aprovado pelo Comitê de Ética em Pesquisa Envolvendo Seres Humanos da Universidade Federal de Minas Gerais (Parecer 963.492 de 25 de fevereiro de 2015).

\section{RESULTADOS}

Os resultados apresentam primeiramente as características da população estudada, e em seguida as categorias analíticas.

\section{Características da população}

Dos 10 terapeutas ocupacionais contatados, um não manifestou interesse em participar do estudo. Assim, os questionários foram enviados a nove profissionais, dos quais cinco retornaram após 30 dias conforme acordado previamente.

Os participantes tinham idade entre 30 e 36 anos, três eram mulheres, com tempo médio de nove anos de formação em Terapia Ocupacional. Os participantes atuavam no INSS como Orientadores Profissionais/ Analistas do Seguro Social inseridos na Reabilitação Profissional. Estavam alocados em três das quatro

(3) Os serviços prestados pelo INSS estruturam-se em unidades descentralizadas que incluem as Superintendências Regionais, as Gerências Executivas, as Agências da Previdência Social, Procuradorias, Auditorias e Corregedorias Regionais ${ }^{15}$. A Região Metropolitana de Belo Horizonte inclui 34 municípios vinculados a quatro Gerências Executivas (Divinópolis, Contagem, Ouro Preto e Belo Horizonte). 
Gerências Executivas da região e, em média, há seis anos na função.

\section{Facilitadores e barreiras ao retorno ao trabalho}

$\mathrm{Da}$ análise dos relatos emergiram três categorias analíticas: Segurados: aspectos pessoais e inter-relacionais; Empresas e INSS: fatores organizacionais e recursos humanos; e Políticas Públicas: capacitação e contratação de trabalhadores.

\section{Segurados: aspectos pessoais e inter-relacionais}

Os participantes apontaram fatores facilitadores do retorno ao trabalho relacionados aos aspectos pessoais dos segurados. Entre eles destacam-se: a "Motivação do trabalhador e uma vivência positiva do processo de adoecimento/acidente" (E5, sexo feminino); a escolaridade elevada "Escolaridade que amplia as possibilidades de inserção em qualificações profissionais" (E2, sexo masculino) e a capacitação profissional "Ter cursos e conhecimentos prévios como informática, que é exigência no mercado de trabalho" (E5, sexo feminino).

Outros facilitadores enfatizados foram: a existência de boa relação entre segurado e a empresa de origem; a existência de vínculo empregatício com empresa de grande porte e que possua cota para pessoas com deficiência e reabilitados do INSS; e trabalhadores jovens.

As barreiras apontadas foram a "Acomodação, principalmente nos casos dos beneficios longos" (E5, sexo feminino) e as experiências laborais pouco diversificadas, ou seja, dos segurados com "Histórico profissional restrito e unidimensional” (E2, sexo masculino).

Além destas, os terapeutas ocupacionais mencionaram: o vínculo de trabalho com empresas terceirizadas e pequenas empresas; a relação permeada por conflitos entre o segurado e a empresa de origem; idade avançada do segurado; características do adoecimento, por exemplo, a condição de saúde e a gravidade do quadro clínico; não aceitação pelo segurado de sua incapacidade; escolaridade muito elevada e falta de conhecimento na área de informática.

\section{Empresas e INSS: fatores organizacionais e recursos humanos}

Os facilitadores do retorno ao trabalho incluíram a "Integração entre equipes do INSS, empresas e outras entidades" e "Parcerias [do INSS] com empresas para realização de treinamento profissional em serviço" (E3, sexo masculino); o investimento dos trabalhadores do INSS "Empenho dos servidores na condução do Programa de Reabilitação Profissional" (E1, sexo feminino) e a integração entre as diferentes áreas do INSS "Possibilidade de discussão dos casos entre a área socioprofissional e a área médica" (E1, sexo feminino).

Por outro lado, os participantes ressaltaram que a ausência de articulação entre as áreas, na perspectiva da interdisciplinaridade, era uma barreira ao retorno ao trabalho visto que leva a centralização das decisões "Prevalência do parecer da área médica em detrimento das demais áreas" (E1, sexo feminino).

A atuação das Gerências em relação aos recursos para a Reabilitação Profissional foi caracterizada como um facilitador quando ocorre o "Bom funcionamento administrativo das Gerências locais que permite a existência dos contratos de aquisição de cursos, órteses e próteses" (E2, sexo masculino); ou como barreira quando não há "boa organização da área de contratação de cursos" (E2, sexo masculino).

Outras barreiras apontadas foram a dificuldade para o estabelecimento de parcerias com empresas e serviços de capacitação "Dificuldade no encaminhamento de segurados para cursos de capacitação profissional" (E1, sexo feminino); empresas que não são comprometidas com a adaptação de postos de trabalho e com a readaptação dos trabalhadores; e, por fim, o cumprimento da legislação que favorece o retorno ao trabalho.

\section{Políticas Públicas: capacitação e contratação de trabalhadores}

Os terapeutas ocupacionais consideraram as políticas públicas como facilitadores e destacaram a Lei $n^{0} 8.213$ de 1991, Planos de Benefícios da Previdência Social - Lei de Cotas. Os participantes ressaltaram ainda o "Programa Nacional de Acesso ao Ensino Técnico em Emprego (PRONATEC) e previsão legal para o fornecimento de recursos para o cumprimento do Programa de Reabilitação Profissional" (E1, sexo feminino). Cabe destacar que enfatizaram a necessidade de garantir o cumprimento destas políticas "Fiscalização constante do Ministério do Trabalho (Lei de Cotas)" (E3, sexo masculino).

Outro facilitador apontado foi o tipo de benefício concedido em decorrência do estabelecimento do nexo causal do acidente/doença com o trabalho que pode facilitar o retorno às atividades "O beneficio [auxílio acidente] é pago como uma forma de indenização em 
função do acidente e, portanto, não impede o cidadão de continuar trabalhando" (E1, sexo feminino).

Por outro lado, as barreiras estão relacionadas a não disponibilização de recursos para a Reabilitação Profissional "Descontinuidade da oferta de recursos para a Reabilitação" (E3, sexo masculino); e a "Dificuldade na efetivação de contratos e convênios para compra de cursos de capacitação profissional" (E1, sexo feminino). Foi também destacada a demora para prestação de serviços na área de saúde "Longo tempo que os segurados permanecem aguardando por procedimentos na área de saúde" (E1, sexo feminino).

Os participantes indicaram ainda que " $A s$ características da região, em especial um mercado de trabalho com boa oferta de empregos e diversidade de ramos produtivos" (E2, sexo masculino) podem ser facilitadores do retorno ao trabalho, por outro lado, a ausência destas características pode ser considerada como uma barreira.

Os terapeutas ocupacionais sugeriram estratégias para enfrentamento das barreiras visando à melhoria do retorno ao trabalho: recomposição, ampliação e qualificação das equipes multiprofissionais; elaboração dos instrumentos de avaliação de melhor qualidade; estabelecimento de convênios em âmbito nacional para facilitar as parcerias locais; maior divulgação da Reabilitação Profissional para a sociedade; aplicação contínua da legislação existente; aprimoramento das relações interministeriais (Previdência Social, Saúde, Educação e Trabalho) e; maior investimento técnico e político na Reabilitação Profissional.

\section{DISCUSSÃO}

Como o retorno ao trabalho é um fenômeno multifatorial, os facilitadores e as barreiras, elencados nas três categorias analíticas, serão discutidos de modo integrado.

Os facilitadores mais destacados pelos terapeutas ocupacionais foram a qualificação e pouca idade do segurado, vínculo com empresa que tenha cota para pessoas com deficiência e/ou reabilitados profissionais, atuação dos servidores da Reabilitação Profissional e das Gerências Executivas na contratação de cursos de capacitação e, por fim, as políticas públicas. Dentre estes a literatura corrobora que a qualificação favorece um desfecho de sucesso no retorno ao trabalho à medida que amplia as oportunidades de emprego e a sua mudança $\mathrm{a}^{10}$, que trabalhadores jovens têm mais facilidade para retornar às atividades ${ }^{11}$ e que a atuação dos servidores são facilitadores do retorno ao trabalho ${ }^{17}$.

Em relação às políticas públicas os participantes apontaram o pagamento do benefício auxílio-acidente, o qual também é referido entre os facilitadores por Cabral et al. ${ }^{10}$. Porém, os participantes indicaram a necessidade de ações efetivas de fiscalização e de articulação intersetorial para assegurar a implementação das políticas.

$\mathrm{O}$ relato dos terapeutas ocupacionais indicou que a motivação dos trabalhadores funciona como um facilitador, porém, quando ausente torna-se uma barreira. Acrescido da falta de motivação tem-se a ausência de interesse das empresas em transformar as situações de trabalho. Saldanha et al. ${ }^{11}$ enfatizam que esta ausência de modificações no posto e na organização do trabalho dificulta o retorno, a permanência do trabalhador e o coloca frente a um novo risco de afastamento. O estudo de Cestari e Carlotto $^{17}$ descreveu a existência de empresas que reinserem o funcionário apenas para cumprir exigências legais. Em algumas situações não há uma preocupação com a compatibilidade entre as funções e as atuais restrições do trabalhador ou são deixados à margem do processo produtivo ${ }^{4,17}$. Além disso, para os trabalhadores que foram afastados do trabalho por motivos relacionados a este, a circunstância do retorno configura um impasse entre a vontade de retornar ao trabalho e a exposição à mesma situação que os levou ao afastamento ${ }^{4}$. De acordo com Cabral et al. ${ }^{10}$, programas de modificação do trabalho favorecem a readaptação do trabalhador, tanto em temporalidade quanto em permanência no trabalho.

Cabe salientar que a análise de posto de trabalho e das atividades a serem desempenhadas pelo trabalhador está prevista nas atribuições dos orientadores profissionais. No entanto, na prática são solicitadas informações a empresa de vínculo sobre a função oferecida e realizado o encaminhamento do trabalhador para um estágio, mas não ocorre uma análise criteriosa da situação real ${ }^{18}$, que se distancia do prescrito frente à necessidade de gerenciamento das variabilidades do trabalho ${ }^{2}$.

O envolvimento de diferentes organizações e atores sociais, com experiências e perspectivas distintas é determinante para o retorno ao trabalho ${ }^{10,11}$. Entretanto os resultados desse estudo apontaram a ausência de articulação entre INSS, empresas e segurados e a centralização de decisões em alguns profissionais, atribuindo a atores isolados a condução de uma problemática complexa. Toldrá et al. ${ }^{4}$ ressaltaram a divisão das atribuições entre peritos e orientadores profissionais no INSS, a qual contribuiu para a fragilização das equipes multiprofissionais. 
Ainda em relação às equipes de trabalho, os participantes sinalizaram a necessidade de sua "recomposição, ampliação e qualificação". Esta estratégia deve orientar-se pela perspectiva da interdisciplinaridade e buscar a construção de espaços para permitir o diálogo e legitimar a participação dos interessados no desenvolvimento de ações coletivas. Assim, os terapeutas ocupacionais podem contribuir para o retorno ao trabalho, incorporando a perspectiva da atividade na compreensão dos indivíduos e das situações produtivas ${ }^{13}$. A percepção do trabalhador afastado, quanto à sua capacidade, a importância e o significado atribuído por ele ao trabalho, deve ser considerada uma vez que influencia seu retorno à atividade ${ }^{3,10}$.

Entre as barreiras que dificultam o retorno ao trabalho, os terapeutas ocupacionais apontaram o baixo grau de escolaridade e as experiências profissionais restritas, resultados corroborados por outros estudos ${ }^{4,5}$, a relação permeada por conflitos entre o trabalhador e a empresa de vínculo, a dificuldade no estabelecimento de parcerias para capacitação e treinamento em serviço, a descontinuidade dos recursos para a Reabilitação Profissional e a demora para prestação de serviços na área de saúde. Esta demora pode ser atribuída, segundo Toldrá et al. ${ }^{4}$, a desarticulação entre os serviços da previdência social e da saúde.

Este estudo apresentou como limite a abrangência restrita a uma Região Metropolitana, e como ponto forte a utilização da metodologia qualitativa que possibilitou ampliar a compreensão, a partir da perspectiva dos terapeutas ocupacionais/orientadores profissionais, sobre os fatores facilitadores e as barreiras para o retorno ao trabalho.

\section{CONSIDERAÇÕES FINAIS}

Considerando que o retorno ao trabalho é um fenômeno dinâmico e multifatorial, este estudo aponta como desafio a articulação de ações de diferentes atores sociais como a previdência social, o sistema de saúde, as empresas e os segurados visando o desenvolvimento de ações que potencializem os facilitadores, modifiquem as barreiras e transformem o cenário atual de retorno e permanência no trabalho.

\section{REFERÊNCIAS}

1. Tersac G, Maggi B. O trabalho e a abordagem ergonômica. In: Daniellou F, coordenador. A ergonomia em busca de seus princípios. Debates epistemológicos. São Paulo: Edgard Blücher; 2004. p.79-104.

2. Guérin F, Laville A, Daniellou F, Duraffourg J, Kerguelen A. Compreender o trabalho para transformá-lo. São Paulo: Editora Blücher; 2001.

3. Cardoso ACM. O trabalho como determinante do processo saúde-doença. Tempo Social, Rev Sociol USP. 2015;27(1):73-94. DOI: 10.1590/0103-207020150110.

4. Toldrá RC, Daldon MTB, Santos MC, Lancman S. Facilitadores e barreiras para o retorno ao trabalho: a experiência de trabalhadores atendidos em um Centro de Referência em Saúde do Trabalhador. Rev Bras Saúde Ocup. 2010;35(121):10-22. DOI: 10.1590/s030376572010000100003 .

5. Bregalda MM, Lopes RE. A atuação dos Terapeutas Ocupacionais no Instituto Nacional do Seguro Social. In: Simonelli AP, Rodrigues DS, organizadores. Saúde e trabalho em debate: velhas questões, novas perspectivas. Brasília: Paralelo; 2013. p.293-321.

6. Brasil. Presidência da República. Casa Civil. Decreto ${ }^{0}$ 3.048 de 06 de maio de 1999. Aprova o regulamento da
Previdência Social, e dá outras providências. Disponível em: http://www.planalto.gov.br/ccivil_03/decreto/d3048.htm.

7. Brasil. Ministério da Previdência Social. Anuário estatístico da Previdência Social 2013. Disponível em: http://www. previdencia.gov.br/dados-abertos/aeps-2013-anuarioestatistico-da-previdencia-social-2013/.

8. Schultz IZ, Stowell AW, Feuerstein M, Gatchel RJ. Models of return to work for musculoskeletal disorders. J Occup Rehabil. 2007;17:327-52. DOI: 10.1007/s10926-007-9071-6.

9. Young AE, Roessler RT, Wasiak R, McPherson KM, van Poppel MNM, Anema JR. A developmental conceptualization of return to work. J Occup Rehabil. 2005;15(4):557-68. DOI: 10.1007/s10926-005-8034-z.

10. Cabral LHA, Sampaio RF, Figueiredo IM, Mancini MC. Fatores associados ao retorno ao trabalho após um trauma de mão: uma abordagem qualiquantitativa. Rev Bras Fisioter. 2010;14(2):149-57. DOI: 10.1590/s141335552010005000004.

11. Saldanha JHS, Pereira APM, Neves RF, Lima MAG. Facilitadores e barreiras de retorno ao trabalho de trabalhadores acometidos por LER/DORT. Rev Bras Saude Ocup. 2013;38(127):122-38. DOI: 10.1590/S030376572013000100014 . 
12. Bregalda MM, Lopes RE. O programa de reabilitação profissional do INSS: apontamentos iniciais a partir de uma experiência. Cad Ter Ocup UFSCar. 2011;19(2):249-61.

13. Lancman S, Daldon MT, Barros J, Gonçalves R, Jardim T. Processos de retorno e permanência no trabalho: elementos estruturantes para a construção de um modelo de ação. In: Simonelli AP, Rodrigues DS, organizadoras. Saúde e trabalho em debate: velhas questões, novas perspectivas. Brasília: Paralelo; 2013. p.135-57.

14. Minayo MCS. O desafio do conhecimento. Pesquisa qualitativa em saúde. 14a ed. São Paulo: Hucitec Editora; 2014.

15. Brasil. Presidência da República. Casa Civil. Decreto $n^{\circ}$ 7556 de 24 de agosto de 2011. Aprova a estrutura regimental e o quadro demonstrativo dos cargos em comissão, das funções gratificadas e das funções comissionadas do Instituto Nacional do Seguro Social - INSS e remaneja cargos em comissão, funções comissionadas e gratificadas. Disponível em: http://www.planalto.gov.br/ccivil_03/ Ato2011-2014/2011/Decreto/D7556.htm.

16. Bardin L. Análise de conteúdo. São Paulo: Edições 70; 2011.

17. Cestari E, Carlotto MS. Reabilitação profissional: o que pensa o trabalhador sobre sua reinserção. Estud Pesqui Psicol. 2012;12(1):93-115.

18. Maeno M, Vilela RAG. Reabilitação profissional no Brasil: elementos para a construção de uma política pública. Rev Bras Saude Ocup. 2010;35(121):87-99. DOI: 10.1590/ S0303-76572010000100010.

Aceito em: 18.04.16

Recebido em: 30.10 .15 